Draft Version OCtOBer 2, 2018

Preprint typeset using $\mathrm{LAT}_{\mathrm{E}} \mathrm{X}$ style emulateapj v. 12/16/11

\title{
A FALLBACK ACCRETION MODEL FOR THE UNUSUAL TYPE II-P SUPERNOVA IPTF14HLS
}

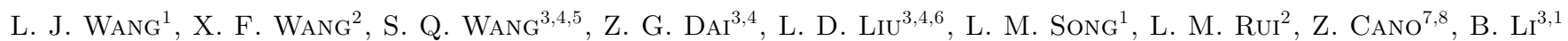 \\ ${ }^{1}$ Astroparticle Physics, Institute of High Energy Physics, Chinese Academy of Sciences, Beijing 100049, China; wanglingjun@ihep.ac.cn \\ ${ }^{2}$ Physics Department and Tsinghua Center for Astrophysics, Tsinghua University, Beijing 100084, China \\ ${ }^{3}$ School of Astronomy and Space Science, Nanjing University, Nanjing 210093, China; dzg@nju.edu.cn \\ ${ }^{4}$ Key Laboratory of Modern Astronomy and Astrophysics (Nanjing University), Ministry of Education, Nanjing 210093, China \\ ${ }^{5}$ Department of Astronomy, University of California, Berkeley, CA 94720-3411, USA \\ ${ }^{6}$ Department of Physics and Astronomy, University of Nevada, Las Vegas, NV 89154, USA \\ ${ }^{7}$ Instituto de Astrofísica de Andalucía (IAA-CSIC), Glorieta de la Astronomía s/n, E-18008, Granada, Spain. and \\ ${ }^{8}$ Juan de la Cierva Fellow. \\ Draft version October 2, 2018
}

\begin{abstract}
The Intermediate Palomar Transient Factory reported the discovery of an unusual type II-P supernova iPTF14hls. Instead of a 100-day plateau as observed for ordinary type II-P supernovae, the light curve of iPTF14hls has at least five distinct peaks, followed by a steep decline at $\sim 1000$ days since discovery. Until 500 days since discovery, the effective temperature of iPTF14hls is roughly constant at $5000-6000 \mathrm{~K}$. In this paper we propose that iPTF14hls is likely powered by intermittent fallback accretion. It is found that the light curve of iPTF14hls can be well fit by the usual $t^{-5 / 3}$ accretion law until $\sim 1000$ days post discovery when the light curve transitions to a steep decline. To account for this steep decline, we suggest a power-law density profile for the late accreted material, rather than the constant profile as appropriated for the $t^{-5 / 3}$ accretion law. Detailed modeling indicates that the total fallback mass is $\sim 0.2 M_{\odot}$, with an ejecta mass $M_{\mathrm{ej}} \simeq 21 M_{\odot}$. We find the third peak of the light curve cannot be well fit by the fallback model, indicating that there could be some extra rapid energy injection. We suggest that this extra energy injection may be a result of a magnetic outburst if the central object is a neutron star. These results indicate that the progenitor of iPTF14hls could be a massive red supergiant.
\end{abstract}

Subject headings: stars: massive — supernovae: general — supernovae: individual (iPTF14hls) X-rays: bursts

\section{INTRODUCTION}

Recently, the discovery of an unusual supernova (SN), iPTF14hls, was reported by Arcavi et al. (2017). iPTF14hls, at a redshift of $z=0.0344$, was first discovered in $R$ band on September 22, 2014 UT (Arcavi et al. 2017). Before its discovery, the position of iPTF14hls was not monitored for approximately 100 days. At beginning, astronomers did not pay much attention to iPTF14hls during its decline in brightness. Intense multiband observations were deployed only when iPTF14hls began to rebrighten after about 100 days since its discovery.

Although identified as a type II-P SN according to its spectroscopic features ( $\mathrm{Li}$ et al. 2015), iPTF14hls is very unique among currently discovered SNe. The light curve of iPTF14hls lasts for more than 1200 days (Sollerman et al. 2018) and has at least five distinct peaks, while an ordinary type II-P SN has a 100-day plateau in brightness. The spectral evolution of iPTF14hls is 10 times slower than typical SNe II-P (Arcavi et al. 2017). The photospheric velocities measured by Fe II $\lambda 5169$ stay at a constant value of $4000 \mathrm{~km} \mathrm{~s}^{-1}$.

Arcavi et al. (2017) discussed several possible theoretical models, e.g., interaction between SN ejecta and circumstellar material (Chevalier 1982; Chevalier \& Fransson 1994; Chugai \& Danziger 1994; Chatzopoulos et al. 2012; Moriya et al. 2013; Wang et al. 2016b, 2017a), spindown of a magnetar (Kasen \& Bildsten 2010; Woosley 2010), fallback accretion onto a black hole (Michel
1988; Dexter \& Kasen 2013), and suggested that the most likely model may be fallback accretion. However, Dessart (2018) proposed that the magnetar model can fit the light curve, while Soker \& Gilkis (2018) explained iPTF14hls as a common-envelope jets SN. Chugai (2018) and Woosley (2018) discussed the models that might explain the light curve and spectral features.

Here we suggest that the multiple peaks in the light curve of iPTF14hls could be powered by intermittent fallback accretion of the SN ejecta. In a successful SN, the material remaining bound could fallback and eventually accrete onto the central object. Accretions onto compact objects (black holes or neutron stars) are usually accompanied by powerful outflows (Mirabel \& Rodríguez 1998; Fender et al. 2004), which can carry away about $10 \%$ of the gravitational binding energy of the accreted material. Such powerful outflows can aid the explosion of the SN, and on the other hand, a fraction of this energy would be thermalized to power a bright light curve (Dexter \& Kasen 2013).

This paper is structured as follows. In Section 2 we describe the fallback accretion process, while in Section 3 the model and fitting results are presented. Finally, we discuss and conclude our results in Section 4.

\section{FALLBACK ACCRETION}

After the explosion of a core-collapse SN, a rebounce outward shock is launched at the base of the central compact core, which further collapses into a neutron star or black hole. This shock imparts a typical kinetic energy 
of $\simeq 10^{51} \mathrm{erg}$ to the still-infalling material and reverses it to move outward. The outward-moving material (ejecta) adjusts itself quickly into a homologous expansion phase, that is, the expansion velocity $v$ of a material element is proportional to its distance $r$ to the central compact object. Although most of the ejecta becomes unbound to the central compact object, a fraction of the ejecta with mass $M_{0}$ is bound and finally falls back (Colgate 1971) and accretes onto the central compact object. Based on some arguments presented in Section 4, hereafter we assume that the remnant of iPTF14hls is a neutron star.

The material accreted at early times comes from the slowly moving inner ejecta. Assuming a power-law density profile of the inner shell of the progenitor star $\rho(r)=\rho_{0}\left(r / r_{0}\right)^{\alpha-3}$, where $\rho_{0}$ is the density of the shell at radius $r_{0}$, the fallback accretion rate is (for $0<\alpha<3$; Quataert \& Kasen 2012; Dexter \& Kasen 2013)

$$
\dot{M}=\frac{8 \pi}{3-\alpha} \frac{\rho_{0} r_{0}^{3}}{t_{0}}\left(\frac{t}{t_{0}}\right)^{\frac{3(\alpha-1)}{3-\alpha}} .
$$

This accretion rate is usually rising because typically $1<$ $\alpha<3$ for inner shells. Here $t_{0}$ is defined as ${ }^{1}$

$$
t_{0}=\left(\frac{\pi \alpha}{32 G \rho_{0}}\right)^{1 / 2} .
$$

This accretion phase will transition to a long-term accretion phase when the expansion velocity $v$ of the bound material is comparable to the escape velocity $v_{\text {esc. In }}$ this case the material can reach a maximum radius $r_{\max }=r_{0}\left(1-v^{2} / v_{\text {esc }}^{2}\right)^{-1}$ (Dexter \& Kasen 2013) and then falls back with a free-fall timescale $t_{\mathrm{ff}}$ (Michel 1988)

$$
\frac{v_{\mathrm{esc}}^{2}-v^{2}}{v_{\mathrm{esc}}^{2}}=\left(\frac{t_{\mathrm{col}}}{t_{\mathrm{ff}}}\right)^{2 / 3}
$$

where $t_{\text {col }}$ is the free-fall collapse time to form $M_{0}$ from material at rest. Assuming a constant density profile, the accretion rate decays according to $t^{-5 / 3}$ (Michel 1988).

At very late phase, instead of a constant profile, the density may be a steep power law, $\rho(r)=\rho_{0}\left(r / r_{0}\right)^{\alpha-3}$ with $\alpha<0$, the enclosed mass is effectively constant, and the accretion rate is (Dexter \& Kasen 2013)

$$
\dot{M}=\frac{8 \pi}{3} \frac{\rho_{0} r_{0}^{3}}{t_{1}}\left(\frac{t}{t_{1}}\right)^{(2 \alpha-3) / 3},
$$

where

$$
t_{1} \equiv \pi\left(\frac{r_{0}^{3}}{8 G M}\right)^{1 / 2}
$$

Because the early rising phase in the light curve of iPTF14hls is missing, we will model the light curve only by the $t^{-5 / 3}$ law and at very late phase $t^{(2 \alpha-3) / 3}$ law with some $\alpha<0$.

Assuming a spherical accretion, Chevalier (1989) and Houck \& Chevalier (1991) studied the structure of the accretion flow that may operate in the famous SN 1987A. To power an SN like iPTF14hls by accretion, the accretion rate (see Section 3 ) should be high (in the range

\footnotetext{
1 Note that this definition of $t_{0}$ is different from that given in Dexter \& Kasen (2013) by an extra factor $\sqrt{\pi \alpha / 8}$.
}

$\left.10^{-4} \lesssim \dot{M}_{\mathrm{ac}} \lesssim 10^{4} M_{\odot} \mathrm{yr}^{-1}\right)$ and the gravitational accretion energy is carried away by neutrinos produced near the neutron star (Chevalier 1989; Houck \& Chevalier 1991). However, this does not mean that the photons in the accretion flow cannot heat the ejecta.

To determine whether the radiation advected with the accretion flow is able to diffuse out, the trapping radius (Katz 1977; Begelman 1978; Flammang 1982; Blondin 1986)

$r_{\mathrm{tr}}=\frac{\dot{M}_{\mathrm{ac}} \kappa}{4 \pi c}=5.5 \times 10^{13}\left(\frac{\dot{M}_{\mathrm{ac}}}{M_{\odot} \mathrm{yr}^{-1}}\right)\left(\frac{\kappa}{0.33 \mathrm{~cm}^{2} \mathrm{~g}^{-1}}\right) \mathrm{cm}$

is defined at which the inwardly advected radiation flux balances the outward diffusion flux. Photons outside this radius can diffuse out and heat the ejecta, while the photons inside this radius are trapped. Because iPTF14hls is hydrogen-rich, here we take the electron Thomson scattering opacity $\kappa=0.33 \mathrm{~cm}^{2} \mathrm{~g}^{-1}$ (e.g., Moriya et al. 2011; Chatzopoulos et al. 2012), which is suitable for fully ionized material with solar metallicity.

The inner regions of the accretion flow achieve supersonic free fall (Chevalier 1989), which, upon reaching the neutron star surface, generates a strong shock moving outward. The energy is mainly stored inside but close to the shock radius. The shock radius $r_{s}$ is determined by neutrino cooling efficiency. Photons inside $r_{s}$ act as potential heating source of the SN. Whether the photons inside $r_{s}$ can diffuse out depends on if the condition $r_{s}>r_{\mathrm{tr}}$ is satisfied. Here we simply assume that this condition is satisfied and leave the justification in Section 4.

At the accretion rate mentioned above, the accretion is super-Eddington. During accretion the infalling material is compressed and becomes hot and geometrically thick because of the inability of the advected photons to escape from the accretion flow. As a result, the accretion is accompanied by powerful outflow (Narayan \& Yi 1994; Blandford \& Begelman 1999; Igumenshchev \& Abramowicz 2000; McKinney et al. 2012), as verified by the observation of ultra-relativistic outflow from a neutron star accreting gas from a companion (Fender et al. 2004). Usually the accretion rate is assumed to be a power-law in radius $\dot{M}(r)=\dot{M}_{\mathrm{fb}}\left(r / r_{\mathrm{fb}}\right)^{s}$ (e.g., Kohri et al. 2005; Dexter \& Kasen 2013), where $\dot{M}_{\mathrm{fb}}$ is the mass accretion rate at the fallback radius $r_{\mathrm{fb}}$, and $0<s<1$. It should be stressed that $\dot{M}_{\mathrm{fb}}$ is not the mass accretion rate onto the compact object because a large fraction of the accretion flow is channeled as an outflow. The net accretion rate $\dot{M}_{\text {ac }}$ onto the neutron star is usually only $\sim 1 \%$ of $\dot{M}_{\mathrm{fb}}$, namely $\dot{M}_{\mathrm{ac}}=\xi \dot{M}_{\mathrm{fb}}$ with $\xi \simeq 0.01$. About $10 \%$ of the accreted matter is converted as radiation energy. Consequently, the accretion energy rate is $\dot{E}_{w}=\epsilon \dot{M}_{\mathrm{fb}} c^{2}$ with $\epsilon \simeq 10^{-3}$ (Dexter \& Kasen 2013).

Because of the existence of powerful outflows, the accretion cannot be strictly spherical. The aspherical accretion may be induced by the spiral modes of the standing accretion shock instability (SASI; Burrows et al. 1995; Janka \& Müller 1996; Blondin et al. 2003; Marek \& Janka 2009; Fernández 2010) or the convection in the pre-collapse envelope (Gilkis \& Soker 2014). Recently, the jet-feedback mechanism (Gilkis et al. 2016) based 
on SASI is suggested to carry out the accretion energy. In this scenario the energy may be carried out by jets accreted near the equatorial plane, as indicated by observations (Fender et al. 2004).

The fallback mass is a function of the compactness of the progenitor stars and explosion energy (Chevalier 1989; Zhang et al. 2008). For loose progenitors with typical explosion energies $\sim 10^{51} \mathrm{erg}$, like red supergiants (RSGs), the fallback mass is usually small, $\lesssim 0.1 M_{\odot}$. However, for more compact progenitors, e.g., blue supergiants, the $\mathrm{H} / \mathrm{He}$ interface triggers the formation of a strong reverse shock, which decelerates the ejecta and enhances the fallback mass significantly. For weak explosions, most of the mass may fall back (Moriya et al. 2010). The metallicity of the progenitor stars influences the mass loss history before explosion and therefore is another factor that impacts the fallback mass. As a result, fallback accretion influences the final mass of the central compact objects. For population III (zero metallicity) stars above $25 M_{\odot}$ and explosion energies less than $1.5 \times 10^{51} \mathrm{erg}$, the central compact objects are more likely black holes (Zhang et al. 2008) because of large amount of fallback. For population I (solar metallicity) stars, black hole production is much less frequent because of large scale mass loss before explosion.

\section{THE MODEL AND FITTING RESULTS}

The accretion outflows not only heat the SN ejecta, but also accelerate the ejecta. We use the method outlined in Wang et al. (2016a) to calculate the light curve and the evolution of the photospheric velocities. In this model the photospheric radius is at the position outside of which the optical depth is equal to $2 / 3$ (Wang et al. 2016a). The acceleration of the ejecta by the energy injection has been taken into account by this model, which assumes a homologous expansion of the SN ejecta, with a homogeneous density distribution. The energy injection from the energy sources, which may be a spinning-down magnetar, ${ }^{56} \mathrm{Ni}$ cascade decay, or fallback accretion, will be trapped by the ejecta. The trapped energy undergoes adiabatic expansion, which accelerates the ejecta according to the following equation (Wang et al. 2016a)

$$
\frac{d E_{K}}{d t}=L_{\mathrm{inp}}-L_{e},
$$

where $L_{\mathrm{inp}}$ is the power trapped by the ejecta, $L_{e}$ is the SN luminosity, and $E_{K}$ is the kinetic energy of the $\mathrm{SN}$. The expansion velocity $v_{\mathrm{sc}}$ (which is approximately equal to the observed photospheric velocity for massive ejecta at early epoch) is calculated according to $E_{K}=$ $3 M_{\mathrm{ej}} v_{\mathrm{sc}}^{2} / 10$ (Arnett 1982), where $M_{\mathrm{ej}}$ is the ejecta mass. A part of the trapped energy diffuses out of the ejecta, resulting in the multiband optical emission of the SN.

To account for the multiple peaks in the light curve of iPTF14hls, we propose that the accretion is episodic. Such episodes are not rare in astrophysics. For example, episodic accretion may be caused by instabilities of disks around protostars (Sakurai et al. 2016; Küffmeier et al. 2018), or by knotty jets in young protostellar disks (Vorobyov et al. 2018).

For fallback accretion, the energy input is

$$
L_{\mathrm{inp}}=\dot{E}_{w}=\epsilon \dot{M}_{\mathrm{fb}}(t) c^{2},
$$

where $\dot{M}_{\mathrm{fb}}(t)$ takes the expression

$$
\dot{M}_{\mathrm{fb}}(t)=\dot{M}_{i}\left(t / t_{i}\right)^{-5 / 3}
$$

during the constant density accretion phase and

$$
\dot{M}_{\mathrm{fb}}(t)=\dot{M}_{i}\left(t / t_{i}\right)^{-(2 \alpha-3) / 3}
$$

for the final power-law density accretion. Here $\dot{M}_{i}$ is the mass fallback rate at time $t_{i}$ when the $i$ th fallback episode begins. To calculate the light curve of an SN powered by fallback accretion, the energy input given by Equation (8) takes the place of the magnetar spinning-down power in the case of a magnetar-powered SN. In both the magnetar-powered case and the accretion-powered case, the energy is assumed to be deposited at the center of the SN ejecta. The photospheric emission is a result of photon diffusion.

In this work we use the bolometric luminosity data of iPTF14hls provided by Sollerman et al. (2018) who extended the observation to more than 1200 days since discovery. We neglect the possible contribution of ${ }^{56} \mathrm{Ni}$ and ${ }^{56} \mathrm{Co}$ to the light curve of iPTF14hls. The SN explosion would have surely synthesized some amount of ${ }^{56} \mathrm{Ni}$. However, because of the finite lifetimes of ${ }^{56} \mathrm{Ni}$ (8.8 days) and ${ }^{56} \mathrm{Co}$ (111.3 days), such contribution is only limited to the first $\sim 100$ days since explosion, which were largely missed by the observation.

The fitting results (solid lines), including the light curve and photospheric velocity evolution, are shown in Figure 1, with the 19 fitting parameters listed in Table 1. It can be found that the fallback rates listed in Table 1 are similar to that given by Moriya et al. (2018), who interpret OGLE-2014-SN-073 as a fallback accretion powered type II supernova. To give a decent fit to the light curve, eight episodes are needed. In Figure 1 we mark $t_{i}$ as vertical blue ticks. It is found that the first seven accretion episodes can be fit by the $t^{-5 / 3}$ law, whereas the last episode $\left(t_{8}\right.$ and $\left.\dot{M}_{8}\right)$ can only be fit by a steep decay with a density power-law index $\alpha \simeq-22$. With this $\alpha$, the late-time light curve decay index is $\sim-15.6$, slightly steeper than that measured by Sollerman et al. (2018), who gave a decay index -13.5 .

The explosion date is $\sim 120$ days before the first observational data point. In Table $1 v_{\mathrm{sc} 0}$ is the initial expansion velocity of the surface of the ejecta. Assuming homologous expansion of the ejecta, the initial explosion energy of this SN is $3 M_{\mathrm{ej}} v_{\mathrm{sc} 0}^{2} / 10=2.2 \times 10^{51} \mathrm{erg}$. This energy can be attributed to neutrino-driven mechanism, which may drive an explosion up to energy $2.5 \times 10^{51}$ erg (Janka et al. 2016; Bollig et al. 2017).

Figure 1 shows that the fallback accretion model gives a reasonably good fit to both the light curve and velocity evolution of iPTF14hls. However, the third peak in the light curve cannot be fitted. Such a steep peak require a very rapid energy release rate. This may suggest some activity in the central compact object. At such late times ( 300 days) since explosion, the energy may be released by a magnetic outburst (Gavriil et al. 2002; Rea et al. 2009,2012 ) if the central object is a neutron star. Indeed, stellar evolution model predicts that a single star with initial masses between $\sim 8$ and $25 M_{\odot}$ will explode as an SN II-P, leaving behind a neutron star remnant (Heger et al. 2003). 
TABLE 1

Best-FitTing PARAMETERs.

\begin{tabular}{|c|c|c|c|c|c|c|c|c|c|c|c|c|c|c|c|c|c|c|}
\hline$M_{\mathrm{ej}}$ & $v_{1}$ & $\dot{M}_{1}$ & $t_{1}$ & $\dot{M}_{2}$ & $t_{2}$ & $\dot{M}_{3}$ & $t_{3}$ & $\dot{M}_{4}$ & $t_{4}$ & $\dot{M}_{5}$ & $t_{5}$ & $\dot{M}_{6}$ & $t_{6}$ & $M_{7}$ & $t_{7}$ & $\dot{M}_{8}$ & $t_{8}$ & $\alpha$ \\
\hline 21 & 4200 & 4.9 & 20 & 0.75 & 186 & .43 & 321 & 0.5 & 380 & 0.4 & 94 & .24 & 550 & .065 & 832 & .043 & 1078 & -22 \\
\hline
\end{tabular}

Notes. $M_{\mathrm{ej}}$ and $v_{\mathrm{sc} 0}$ are in units of $M_{\odot}$ and $\mathrm{km} \mathrm{s}^{-1}$, respectively. The accretion rates $\dot{M}_{i}$ at fallback radius are in units of $10^{-8} M_{\odot} \mathrm{s}^{-1}$, while $t_{i}$ are in units of days since SN explosion. In this fit we fixed $\kappa=0.33 \mathrm{~cm}^{2} \mathrm{~g}^{-1}$. Because of the lack of observational data between the third and fourth peaks, $t_{4}$ cannot be accurately constrained, so is $t_{1}$ because of the missing of observational data around the first peak. The first seven accretion episodes can be fit by the $t^{-5 / 3}$ law, whereas the last episode $\left(t_{8}\right.$ and $\left.\dot{M}_{8}\right)$ can only be fit by a steep decay with a density power-law index $\alpha$.
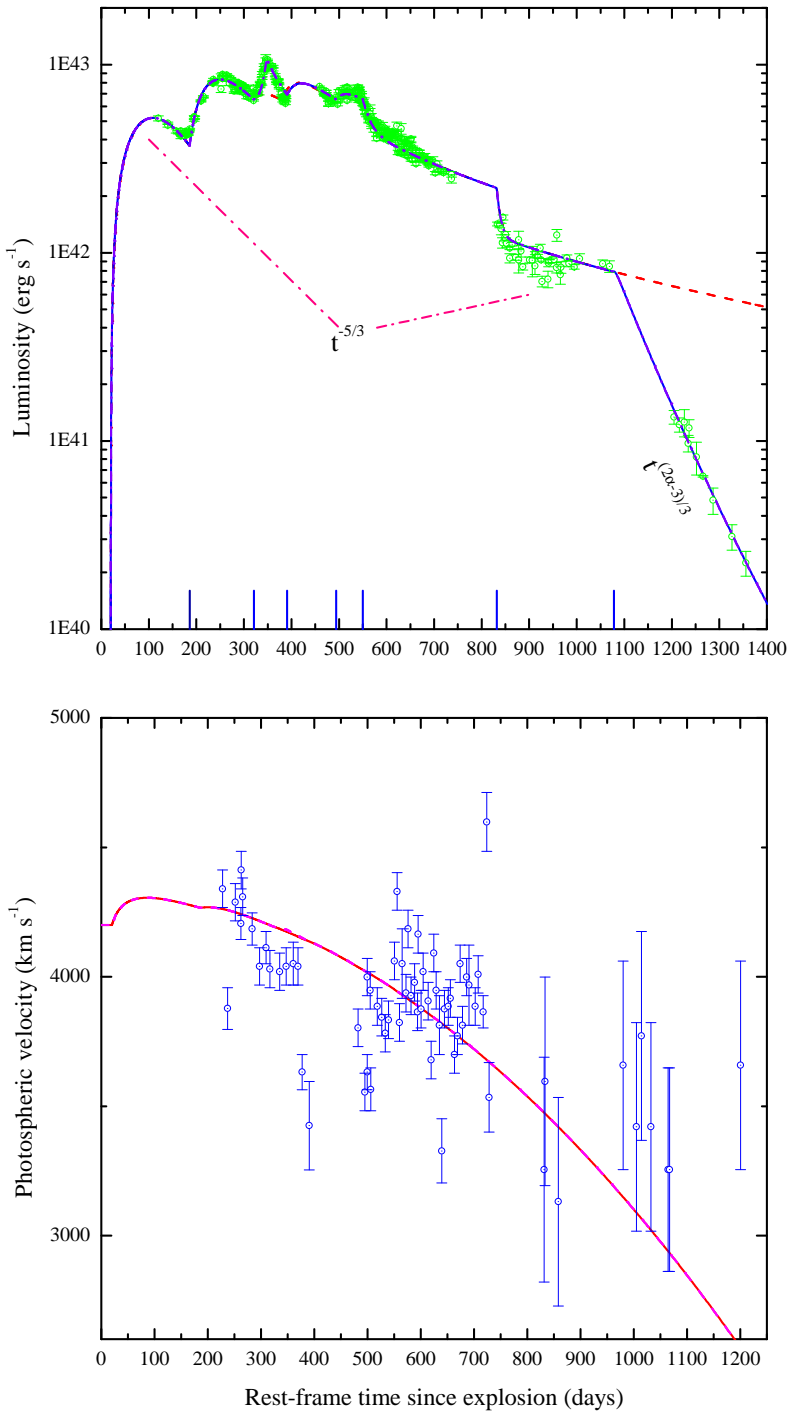

FIG. 1. - Light curve (top panel) and photospheric velocity (bottom panel) of iPTF14hls reproduced by the fallback accretion model (solid lines). The dashed lines are the modeling results including the energy injection from an outburst. The red dashed line in the upper panel assumes a $t^{-5 / 3}$ accretion rate, which fails to fit the data after $\sim 1100$ days since explosion. This is why a late-time steep decline has been introduced here, as depicted by the solid blue line.

To quantify the outburst power, we assume a powerlaw injection

$$
L_{\text {rise }}=L_{\mathrm{pk}}\left(\frac{t-t_{\text {start }}}{t_{\text {rise }}}\right)^{n}
$$

followed by a rapid shutoff of the outburst

$$
L_{\text {fall }}=L_{\mathrm{pk}}\left(\frac{t_{\text {shutoff }}-t}{t_{\text {fall }}}\right) .
$$

Here $t_{\text {start }}$ and $t_{\text {shutoff }}$ are the times at which the outburst begins and ends, respectively; $t_{\text {rise }}$ and $t_{\text {fall }}$ are the durations for the rise and fall of the outburst, respectively. Obviously, $t_{\text {shutoff }}-t_{\text {start }}=t_{\text {rise }}+t_{\text {fall }}$. We set $n=3$ in Equation (11) during the fitting. This power exponent does not result from the fitting constraints but was appropriately selected. To give a good fit to the light curve, we found $2 \lesssim n \lesssim 5$. The 5 fitting parameters for the outburst are listed in Table 2, with the resulting light curve depicted in Figure 1 as dashed lines. Here we choose another set of values for $t_{4}$ and $\dot{M}_{4}$ (see Section 4 for some discussion). It can be seen that the model including a magnetic outburst can fit the light curve very closely.

TABLE 2

FitTing PARAMETERS FOR THE OUTBURST.

\begin{tabular}{ccccc}
\hline \hline$t_{\text {start }}$ & $t_{\text {rise }}$ & $t_{\text {fall }}$ & $L_{\mathrm{pk}}$ & $n$ \\
\hline (days) & $($ days $)$ & $($ days $)$ & $\left(\mathrm{erg} \mathrm{s}^{-1}\right)$ & \\
\hline 318 & 29.2 & 0.8 & $1.7 \times 10^{43}$ & 3 \\
\hline
\end{tabular}

From Table 2 we see that the outburst lasted for $\sim 30$ days, and released $1.1 \times 10^{49} \mathrm{erg}$ in total. This is in accordance with observations, which show that some $\mathrm{X}$-ray pulsars may experience sporadic giant X-ray outbursts lasting weeks to years followed by a long-term quiescence (Gavriil et al. 2002; Kaspi et al. 2003; Rea et al. 2012; Cusumano et al. 2016).

One intriguing feature of iPTF14hls is that the radius derived by the Fe II $\lambda 5169$ expansion velocity times the elapsed rest-frame time is not equal to the radius determined by blackbody fits (see Figure 2). Spectrum measurements of Fe II $\lambda 5169$ indicate that iron expands at roughly a constant velocity $\sim 4000 \mathrm{~km} \mathrm{~s}^{-1}$, from which the so-called line-forming radius can be derived (pink points in Figure 2). This radius is, however, much larger than the blackbody-determined radius (blue circles in Figure 2). As can be seen from Figure 2, the photospheric radius predicted by this model closely follows the line-forming radius. We discuss this feature in Section 4.

\section{DISCUSSION AND CONCLUSIONS}

To date, many SNe were found to be double-peaked (Arnett et al. 1989; Richmond et al. 1994; Mazzali et al. 2008; Nicholl et al. 2015; Nicholl \& Smartt 2016), in 


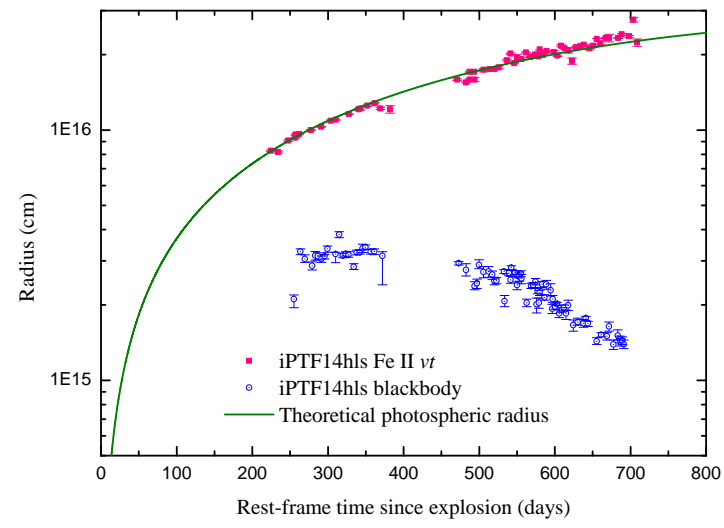

FIG. 2.- Fit (solid green line) to the photospheric radius of iPTF14hls estimated (1) using blackbody fits to the broad-band $B V g i$ photometry (blue) and (2) using the expansion velocities of Fe II $\lambda 5169$ times the elapsed rest-frame time $(v t)$ since explosion (pink). The data are taken from Arcavi et al. (2017), but rescaled to times since explosion, rather than since discovery.

which case the first short-lived peak has been attributed to shock cooling (Piro 2015; Vreeswijk et al. 2017; Wang et al. 2017c), although sometimes the cooling peak could merge with the second main peak (Wang et al. 2017a). The first peak in the light curve of iPTF14hls is unlikely the result of shock cooling because that would require a very massive and extended envelope.

Because of the lack of observational data between the third and fourth peaks of the light curve, and also the missing of observational data around the first peak, $t_{1}$ and $t_{4}$ in Table 1 cannot be accurately constrained. As a demonstration of this uncertainty, in Figure 1 we choose different $t_{4}$ for the solid and dashed curves. However, as can be seen from Figure 1, an earlier $t_{4}$, as depicted by the dashed curve, is preferred because of the lightcurve decline rate between $t_{4}$ and $t_{5}$. For the dashed line (the curve including the energy injection of a magnetic outburst), we choose $t_{4}=391$ days and $\dot{M}_{4}=0.49 \times$ $10^{-8} M_{\odot} \mathrm{s}^{-1}$.

The most likely progenitor of iPTF14hls is a red supergiant since observations have demonstrated that the progenitors of several type II-P SNe are RSGs (e.g., Smartt 2009; Davies 2017; Van Dyk 2017; Huang et al. 2018). This is consistent with the fact that the total fallback mass is about $\sim 0.2 M_{\odot}$, as expected for a RSG progenitor (Chevalier 1989; Dexter \& Kasen 2013). The ejecta mass, $M_{\mathrm{ej}} \simeq 21 M_{\odot}$, is also consistent with a RSG (Davies \& Beasor 2018), though at the high end of the distribution of the SNe II-P ejecta masses.

The remnant of a RSG explosion is believed to be a neutron star. This is consistent with the study of remnant mass distribution of massive star explosions (Zhang et al. 2008). iPTF14hls occurred on the outskirts of a low-mass star-forming galaxy, indicating low metallicity (Arcavi et al. 2017). For population III star explosions with progenitor mass larger than $25 M_{\odot}$ and explosion energies less than $1.5 \times 10^{51} \mathrm{erg}$, black holes are more frequent outcome. The progenitor mass of iPTF14hls, $22 M_{\odot}$ (the sum of $M_{\mathrm{ej}}$ and remnant mass, which is assumed to be $\sim 1 M_{\odot}$ ), combined with its explosion energy $2.2 \times 10^{51} \mathrm{erg}$, indicates that the remnant of iPTF14hls is more likely a neutron star. On the other hand, despite the possible low metallicity of iPTF14hls, Arcavi et al. (2017) estimated a metallicity of $0.5 Z_{\odot}$, which is more compatible with a population I star explosion. In this case the production of a black hole remnant is highly suppressed (Zhang et al. 2008).

The production of a neutron star remnant is also partially supported by the need to fit the third peak of the light curve by a magnetic outburst. Inspection of Table 1 shows that the mass fallback rates $\dot{M}_{i}$ decrease monotonically, as expected. However, $\dot{M}_{1}$ is much larger than $\dot{M}_{2}$. This is in sharp contrast to the mass fallback rates that follow. $\dot{M}_{1}$ could be reduced if there is some contribution from the energy injection of the neutron star. We have neglected the contribution of ${ }^{56} \mathrm{Ni}$ and ${ }^{56} \mathrm{Co}$ because their contribution is short-lived, but the contribution of a neutron star (or magnetar) could be long-lived (Kasen \& Bildsten 2010; Woosley 2010; Inserra et al. 2013; Nicholl et al. 2014; Metzger et al. 2015; Wang et al. 2015, 2017b; Dai et al. 2016; Liu et al. 2017).

To examine the properties (dipole magnetic field $B_{p}$, and initial spin period $P_{0}$ ) of the neutron star, we set (somewhat arbitrarily) $\dot{M}_{1}=1.0 \times 10^{-8} M_{\odot} \mathrm{s}^{-1}$ and found $P_{0} \simeq 8 \mathrm{~ms}, B_{p} \simeq 5 \times 10^{14} \mathrm{G}$. With these parameters, the first peak can be closely fitted while the remaining peaks are affected negligibly by the contribution of the magnetar. We will not show the fitting results by this model because the resulting light curve closely follows the curves presented in Figure 1. It is found that $P_{0}$ cannot be too large, say $P_{0} \gtrsim 10 \mathrm{~ms}$, because in that case the magnetar would contribute too much at late times so that the late-time light curve deviates from the $t^{-5 / 3}$ law. We note that the above constraints on $P_{0}$ and $B_{p}$ should not be taken seriously because they are degenerated with $\dot{M}_{1}$.

As we said, the third peak cannot be explained by fallback accretion, and magnetic activity is therefore proposed as its energy source. For the fallback rate at the third peak, $\dot{M}_{\mathrm{fb}} \simeq 0.4 \times 10^{-8} M_{\odot} \mathrm{s}^{-1}$, the corresponding accretion rate $\dot{M}_{\mathrm{ac}}=\xi \dot{M}_{\mathrm{fb}} \simeq 1 \times 10^{-3} M_{\odot} \mathrm{yr}^{-1}$ indicates a trapping radius $r_{\mathrm{tr}} \simeq 7 \times 10^{10} \mathrm{~cm}$. For the energy in the magnetic activity to diffuse out, the dissipation radius $r_{\text {act }}$ of the magnetic energy should be larger than $r_{\mathrm{tr}}$, namely $r_{\mathrm{act}}>r_{\mathrm{tr}}$. To estimate $r_{\mathrm{act}}$, let us first assume a spherical accretion. The balance of stellar wind pressure $L / 4 \pi r^{2} c$ with the ram pressure $\rho v^{2}=\left(\dot{M}_{\mathrm{ac}} / 4 \pi\right)\left(2 G M / r^{5}\right)^{1 / 2}$ of the infalling material gives a radius

$$
r_{b}=2 G M\left(\frac{\dot{M}_{\mathrm{ac}} c}{L}\right)^{2} \simeq 1.3 \times 10^{12} \mathrm{~cm},
$$

where the typical value of pulsar luminosity $L=1 \times$ $10^{41} \mathrm{erg} \mathrm{s}^{-1}$ at the time of the third peak, the neutron star mass $M=1.4 M_{\odot}$, accretion rate $\dot{M}_{\mathrm{ac}}=\xi \dot{M}_{\mathrm{fb}}=$ $0.01 \times 10^{-8} M_{\odot} \mathrm{s}^{-1}$ have been substituted. This balance is unstable. During the accretion phase, mater falls within $r_{b}$. During the magnetic outburst phase, the central neutron star inflates a bubble, known as pulsar wind nebula (PWN), whose radius $r_{\text {act }}$ is much larger than $r_{b}$, which is also larger than $r_{\mathrm{tr}}$. As a result, the magnetic energy 
stored within the PWN can diffuse out of the accretion flow.

During the normal accretion phase the balance between the magnetic pressure and the accretion ram pressure cannot be maintained and the material falls well within $r_{b}$. The magnetic outburst is usually triggered by some instability of the PWN. The energy released by the spinning-down pulsar does not lose immediately as radiation. It is estimated that about half of the energy lost by Crab $\left(\sim 1.8 \times 10^{49} \mathrm{erg}\right)$ is still resident within the synchrotron nebula (Hester 2008). This energy is very close to the energy assumed here to power the third peak of iPTF14hls. The magnetic activity may interplay with and even quench the accretion. If the accretion is quenched by the magnetic activity, then the third peak is purely powered by the magnetic activity. This argument also applies to the first peak where a magnetar spin-down was proposed to contribute most part of the SN luminosity.

For a rapidly spinning magnetar, outside of the light cylinder, $R_{c}=c P /(2 \pi)=3.8 \times 10^{7}(P / 8 \mathrm{~ms}) \mathrm{cm}$, the magnetic field lines of the magnetar cannot corotate with the magnetar (Shapiro \& Teukolsky 1983), and therefore the field lines in the PWN wind tightly to form a spindle nebula (see Figure 3 of Hester 2008), whose toroidal field is amplified significantly and much stronger than its poloidal field. The lower bound of $r_{\text {act }}$ given by Equation (13) yields an upper limit of the strength of the magnetic field within the PWN, $B_{\mathrm{PWN}}<\left(8 \pi E_{\text {burst }} / V\right)^{1 / 2}=5 \times$ $10^{6} \mathrm{G}$, where $V=4 \pi r_{b}^{3} / 3$ is the lower limit of the volume of the PWN, and $E_{\text {burst }} \sim 1.1 \times 10^{49} \mathrm{erg}$ is the magnetic outburst energy.

The total magnetic outburst energy, $E_{\text {burst }} \sim 1.1 \times$ $10^{49} \mathrm{erg}$, should be accumulated during the first 320 days before the third peak. This requires an average energy injection rate $4 \times 10^{41} \mathrm{erg} \mathrm{s}^{-1}$ during this period. $^{2}$ We found that with the magnetar parameters $\left(P_{0} \simeq 8 \mathrm{~ms}, B_{p} \simeq 5 \times 10^{14} \mathrm{G}\right)$ listed above to explain the first peak of iPTF14hls, it is just right to give such an average energy injection rate. At day 1400 since explosion, the magnetar's energy injection rate declines to $1 \times 10^{40} \mathrm{erg} \mathrm{s}^{-1}$, which is lower than but comparable to the observed luminosity of iPTF14hls. After 1400 days since explosion, the magnetar's energy injection rate dominates over the accretion energy injection rate. Therefore the suggestion of magnetic outburst scenario for the third peak can be falsified if future observation does not reveal a flattening of the luminosity of iPTF14hls after 1400 days.

As mentioned in Section 3, the initial explosion energy $\left(2.2 \times 10^{51} \mathrm{erg}\right)$ of this SN can be attributed to neutrino heating. However, for an SN that is powered by fallback accretion, the explosion energy may also be partially provided by accretion, especially the recently proposed jet-feedback mechanism (Gilkis et al. 2016; Soker 2016, 2017).

We mentioned in Section 1 that for spherical accretion, the photons behind the accretion shock can diffuse out and heat the ejecta if the condition $r_{s}>r_{\text {tr }}$ is fulfilled. Assuming a power-law neutrino cooling function,

\footnotetext{
2 The true energy injection rate should be somewhat higher because a part of the injected energy will leak out of the PWN.
}

the shock position takes the approximate form (Houck \& Chevalier 1991)

$$
r_{s}=1.6 \times 10^{8}\left(\frac{\dot{M}_{\mathrm{ac}}}{M_{\odot} \mathrm{yr}^{-1}}\right)^{-2 / 5} \mathrm{~cm} .
$$

With the peak accretion rate $\dot{M}_{\mathrm{ac}} \simeq 10^{-3} M_{\odot} \mathrm{yr}^{-1}$, the above equation gives $r_{s}=2.5 \times 10^{9} \mathrm{~cm}$, which is at its face value smaller than the trapping radius $r_{\mathrm{tr}} \simeq 7 \times 10^{10} \mathrm{~cm}$. However, the above estimate of $r_{s}$ should be treated as a lower limit because of the uncertainties in neutrino cooling function and relativistic corrections (Houck \& Chevalier 1991). It is actually found that the condition $r_{s}>r_{\text {tr }}$ is satisfied when $\dot{M}_{\mathrm{ac}} \lesssim 10^{-3} M_{\odot} \mathrm{yr}^{-1}$ (Houck \& Chevalier 1991), which is the case for the accretion episodes listed in Table 1, except for the first accretion episode because for the fallback rate $\dot{M}_{\mathrm{fb}} \simeq 0.7 \times 10^{-8} M_{\odot} \mathrm{s}^{-1}$ (see Table 1), the accretion rate is $\dot{M}_{\mathrm{ac}}=\xi \dot{M}_{\mathrm{fb}} \simeq$ $2 \times 10^{-3} M_{\odot} \mathrm{yr}^{-1}$. For the scenario proposed in this paper to be valid, a magnetar energy input is necessary for the first accretion episode. This also strengthens the hypothesis of the formation of a magnetar in this SN explosion.

With the condition $r_{s}>r_{\mathrm{tr}} \simeq 7 \times 10^{10} \mathrm{~cm}$, the shock radius $r_{s}$ is about four orders of magnitude larger than the Schwarzschild radius and it seems unlikely to convert $10 \%$ of the gravitational binding energy of the accreted material into radiation energy. However, the exact accretion process is that the accretion flow reaches at the neutron star surface, where shock is formed and energy is advected along with the outmoving shock and carried far away from the neutron star surface. The shock eventually stops at $r_{s}$ because of efficient neutrino cooling. During this process, a significant fraction (approximately $10 \%$ ) of the gravitational binding energy is converted into shock energy. Note that the fallback energy conversion factor $\epsilon \simeq 10^{-3}$ consists of two factors: the ratio of accretion rate to the fallback rate $\left(\xi=\dot{M}_{\mathrm{ac}} / \dot{M}_{\mathrm{fb}} \simeq 0.01\right)$ and the conversion efficiency (10\%) of the gravitational binding energy.

To account for the late steep decline of the light curve, we suggest that accreted material has a power-law density profile with $\alpha=-22$ at late time. If we adopt the light curve decay index -13.5 , as measured by Sollerman et al. (2018), we found $\alpha=-18$. This density profile is very steep and may be formed by the interaction between the bound and unbound material. Future numerical simulations are encouraged to test this hypothesis.

We propose that the third, brightest peak is mainly powered by a magnetic outburst. Such outburst is usually accompanied by X-ray emission, which is however not detected (Arcavi et al. 2017). The nondetection of $\mathrm{X}$-ray emission can be understood by considering the optical depth of the ejecta in the X-ray band

$$
\begin{aligned}
\tau_{X} & =\frac{3 \kappa_{X} M_{\mathrm{ej}}}{4 \pi v_{\mathrm{sc}}^{2} t^{2}} \\
& =20\left(\frac{M_{\mathrm{ej}}}{21 M_{\odot}} \frac{\kappa_{X}}{0.33 \mathrm{~cm}^{2} \mathrm{~g}^{-1}}\right)\left(\frac{v_{\mathrm{sc}}}{4300 \mathrm{~km} \mathrm{~s}^{-1}}\right)^{-2}\left(\frac{t}{350 \text { days }}\right)^{-2}(15)
\end{aligned}
$$

where the values of X-ray opacity $\kappa_{X}$, SN expansion velocity $v_{\mathrm{sc}}$, and the time since explosion $t$ have been sub- 
stituted. Here $v_{\mathrm{sc}}$ is slightly larger than the initial expansion velocity $v_{\mathrm{sc} 0}$ because of the energy injection. We see that at the time the third peak was observed, the ejecta are still opaque to X-rays. In the above estimate, $\kappa_{X}$ is taken to be the same as $\kappa$, that is, the electron Thomson scattering opacity. This should be a lower limit to the true X-ray opacity because other heavier elements could make a significant contribution to $\kappa_{X}$.

Despite the nondetection of X-ray emission, the detection of $\gamma$-ray emission, temporally and positionally consistent with iPTF14hls, in the energy band between 0.2 and $500 \mathrm{GeV}$ was report by Yuan et al. (2018). The $\gamma$-ray source appears $\sim 300$ days after the first optical detection of iPTF14hls and is still detectable up to $\sim 850$ days. Translated to the time since SN explosion in our model, the $\gamma$-ray source appears $\sim 420$ days to $\sim 970$ days. According to Equation (15), assuming a lower limit to $\gamma$ ray opacity $\kappa_{\gamma}=0.33 \mathrm{~cm}^{2} \mathrm{~g}^{-1},{ }^{3}$ the $\mathrm{SN}$ ejecta are still opaque to $\gamma$-ray emission at time $t \sim 970$ days. Therefore, in our model the $\gamma$-ray emission cannot come from the deep interior of the SN ejecta.

This $\gamma$-ray emission may alternatively result from the interaction between the ejecta and circumstellar medium (CSM) or produced by a blazar because there is a blazar candidate within the error circle of the $\gamma$-ray source (Yuan et al. 2018). Late-time observation of iPTF14hls revealed narrow $\mathrm{H} \alpha$ emission (Andrews \& Smith 2018), which may be evidence for circumstellar interaction where unshocked circumstellar material is ionized by the shock emission and recombines. However, such evidence for interaction only appears at 3 years after the first optical detection of iPTF14hls. The interaction origin of the $\gamma$-ray emission is also in tension with the aforementioned nondetection of X-ray and radio emission. Sollerman et al. (2018) argue that the narrow $\mathrm{H} \alpha$ emission may come from $\mathrm{H}$ II region which is located just at the SN position. Because the $\gamma$-ray association with iPTF14hls is only tentative, we consider it more likely that the $\gamma$-ray emission is produced by the blazar.

Observations indicate that the photospheric radius of iPTF14hls is quite different from the line-forming region. Arcavi et al. (2017) estimate the latter at position of $v t$, where $v$ is the $\mathrm{SN}$ expansion velocity. Although the photospheric radius of an SN recedes as the SN expands and inner material is observed, the large ejecta mass, as inferred from light curve modeling, implies that the photospheric recession should be negligible during the first two years since its discovery. The discrepancy of these two radii might be linked to the existence of persistent Balmer series P Cygni lines observed in the spectra of iPTF14hls (Arcavi et al. 2017). The presence of P-Cygni profiles betrays the existence of a stellar wind, as observed in Wolf-Rayet stars (Willis 1982) and luminous blue variables (Israelian \& de Groot 1999). We suggest that this wind is far above the photosphere and is responsible for the spectral lines.

The rarity of iPTF14hls among SNe II-P may be understood because of its extreme ejecta mass. This large ejecta mass may also account for the reason why so much mass falls back so as to give a multi-peaked light curve.

In summary, iPTF14hls can be explained by the episodic fallback accretion model. ${ }^{4}$ The fitting parameters suggest a RSG as the progenitor. Although the central object cannot be identified, the rapid third peak and other considerations might indicate the formation of a neutron star that experienced a magnetic outburst lasting for $\sim 30$ days with a total burst energy $1.1 \times 10^{49} \mathrm{erg}$.

We thank Iair Arcavi and Jesper Sollerman for providing us the observational data. We also thank the anonymous referee for helpful comments. This work is supported by the National Program on Key Research and Development Project of China (Grant Nos. 2016YFA0400801 and 2017YFA0402600), National Basic Research Program of China ("973" Program, Grant No. 2014CB845800) and the National Natural Science Foundation of China (Grant Nos. 11573014, 11533033, 11673006). X. Wang is supported by the National Natural Science Foundation of China (NSFC grants 11325313 and 11633002), and the National Program on Key Research and Development Project (grant no. 2016YFA0400803). S.Q.W. and L.D.L. are also supported by China Scholarship Program to conduct research at U.C. Berkeley and UNLV, respectively.

\section{REFERENCES}

Andrews, J. E., \& Smith, N. 2018, MNRAS, 477, 74

Arcavi, I., Howell1, D. A., Kasen, D., et al. 2017, Natur, 551, 210

Arnett, W. D. 1982, ApJ, 253, 785

Arnett, W. D., Bahcall, J. N., Kirshner, R. P., \& Woosley, S. E. 1989, ARA\&A, 27, 629

Begelman, M. C. 1978, MNRAS, 184, 53

Blandford, R. D., \& Begelman, M. C. 1999, MNRAS, 303, L1

Blondin, J. M. 1986, ApJ, 308, 755

Blondin, J. M., Mezzacappa, A., \& DeMarino, C. 2003, ApJ, 584, 971

Bollig, R., Janka, H.-T., Lohs, A., et al. 2017, PhRvL, 119, 242702

Burrows, A., Hayes, J., \& Fryxell, B. A. 1995, ApJ, 450, 830

Chatzopoulos, E., Wheeler, J. C., \& Vinko, J. 2012, ApJ, 746, 121

Chevalier, R. A. 1982, ApJ, 258, 790

${ }^{3}$ At late stage, the $\gamma$-ray photons come from ${ }^{56}$ Co decay with typical energy $\sim 1 \mathrm{MeV}$. At this energy, the atomic scattering opacity (Kotera et al. 2013) of a type II SN is approximately equal to the electron Thomson scattering opacity.

${ }^{4}$ Alternatively, the CSM interaction is also a plausible model. Liu et al. (2018) propose a multiple ejecta-CSM interaction model and employed it to model multi-peak SNe iPTF15esb and iPTF13dcc.
Chevalier, R. A. 1989, ApJ, 346, 847

Chevalier, R. A., \& Fransson, C. 1994, ApJ, 420, 268

Chugai, N. N. 2018, AstL, 44, 370

Chugai, N. N., \& Danziger, I. J. 1994, MNRAS, 268, 173

Colgate, S. A. 1971, ApJ, 163, 221

Cusumano, G., La Parola, V., D'Aì, A., et al. 2016, MNRAS, 460, L99

Dai, Z. G., Wang, S. Q., Wang, J. S., Wang, L. J., Yu, Y. W.

2016, ApJ, 817, 132

Davies, B. 2017, RSPTA, 375, 20160270

Davies, B., \& Beasor, E. R. 2018, MNRAS, 474, 2116

Dessart, L. 2018, A\&A, 610, L10

Dexter, J., \& Kasen, D. 2013, ApJ, 772, 30

Fender, R., Wu, K., Johnston, H., et al. 2004, Natur, 427, 222

Fernández, R. 2010, ApJ, 725, 1563

Flammang, R. A. 1982, MNRAS, 199, 833

Gavriil, F. P., Kaspi, V. M., \& Woods, P. M. 2002, Natur, 419, 142

Gilkis, A., \& Soker, N. 2014, MNRAS, 439, 4011

Gilkis, A., Soker, N., \& Papish, O. 2016, ApJ, 826, 178

Heger, A., Fryer, C. L., Woosley, S. E., et al. 2003, ApJ, 591, 288

Hester, J. J. 2008, ARA\&A, 46, 127

Houck, J. C., \& Chevalier, R. A. 1991, ApJ, 376, 234 
Huang, F., Wang, X.-F., Hosseinzadeh, G., et al. 2018, MNRAS, 475,3959

Igumenshchev, I. V., \& Abramowicz, M. A. 2000, ApJS, 130, 463 Inserra, C., Smartt, S. J., Jerkstrand, A., et al. 2013, ApJ, 770, 128

Israelian, G., \& de Groot, M. 1999, SSRv, 90, 493

Janka, H.-T., Melson, T., \& Summa, A. 2016, ARNPS, 66, 341

Janka, H.-T., \& Müller, E. 1996, A\&A, 306, 167

Kasen, D., \& Bildsten, L. 2010, ApJ, 717, 245

Kaspi, V. M., Gavriil, F. P., Woods, P. M., et al. 2003, ApJL, 588, L93

Katz, J. I. 1977, ApJ, 215, 265

Kohri, K., Narayan, R., \& Piran, T. 2005, ApJ, 629, 341

Kotera, K., Phinney, E. S., \& Olinto, A. V. 2013, MNRAS, 432, 3228

Küffmeier, M., Frimann, S., Jensen, S. S., \& Haugbølle, T. 2018, MNRAS, 475,2642

Li, W. X., Wang, X. F., \& Zhang, T. M. 2015, ATel, 6898

Liu, L. D., Wang, L. J., Wang, S. Q., Dai, Z. G. 2018, ApJ, 856, 59

Liu, L. D., Wang, S. Q., Wang, L. J., et al. 2017, ApJ, 842, 26

Marek, A., \& Janka, H.-T. 2009, ApJ, 694, 664

Mazzali, P. A., Valenti, S., Della Valle, M., et al. 2008, Sci, 321, 1185

McKinney, J. C., Tchekhovskoy, A., \& Blandford, R. D. 2012, MNRAS, 423, 3083

Metzger, B. D., Margalit, B., Kasen, D., Quataert, E. 2015, MNRAS, 454, 3311

Michel, F. C. 1988, Natur, 333, 644

Mirabel, I. F., \& Rodríguez, L. F. 1998, Natur, 392, 673

Moriya, T. J., Maeda, K., Taddia, F., et al. 2013, MNRAS, 435, 1520

Moriya, T. J., Terreran, G., \& Blinnikov, S. I. 2018, MNRAS, 475, L11

Moriya, T., Tominaga, N., Blinnikov, S. I., Baklanov, P. V., \& Sorokina, E. I. 2011, MNRAS, 415, 199

Moriya, T., Tominaga, N., Tanaka, M., et al. 2010, ApJ, 719, 1445

Narayan, R., \& Yi, I. 1994, ApJL, 428, L13

Nicholl, M., \& Smartt, S. J. 2016, MNRAS, 457, L79
Nicholl, M., Smartt, S. J., Jerkstrand, A., et al. 2014, MNRAS, 444,2096

Nicholl, M., Smartt, S. J., Jerkstrand, A., et al. 2015, ApJL, 807, L18

Piro, A. L. 2015, ApJL, 808, L51

Quataert, E., \& Kasen, D. 2012, MNRAS, 419, L1

Rea, N., Israel, G. L., Esposito, P., et al. 2012, ApJ, 754, 27

Rea, N., Israel, G. L., Turolla, R., et al. 2009, MNRAS, 396, 2419

Richmond, M. W., Treffers, R. R., Filippenko, A. V., et al. 1994, AJ, 107, 1022

Sakurai, Y., Vorobyov, E. I., Hosokawa, T., et al. 2016, MNRAS, 459, 1137

Shapiro, S. L., \& Teukolsky, S. A. 1983, Black Holes, White Dwarfs, and Neutron Stars: The Physics of Compact Objects (New York: Wiley)

Smartt, S. J. 2009, ARA\&A, 47, 63

Soker, N. 2016, NewAR, 75, 1

Soker, N. 2017, RAA, 17, 113

Soker, N., \& Gilkis, A. 2018, MNRAS, 475, 1198

Sollerman, J., Taddia, F., Arcavi, I., et al. 2018, arXiv:1806.10001

Van Dyk, S. D. 2017, RSPTA, 375, 20160277

Vorobyov, E., Elbakyan, V., Plunkett, A., et al. 2018, A\&A, 613, A18

Vreeswijk, P. M., Leloudas, G., Gal-Yam, A., et al. 2017, ApJ, 835,58

Wang, L. J., Wang, S. Q., Dai, Z. G., et al. 2016a, ApJ, 821, 22

Wang, L. J., Wang, X. F., Cano, Z., et al. 2017a, ApJ, submitted (arXiv:1712.07359)

Wang, L. J., Yu, H., Liu, L. D., et al. 2017b, ApJ, 837, 128

Wang, S. Q., Cano, Z., Wang, L. J., et al. 2017c, ApJ, 850, 148

Wang, S. Q., Liu, L. D., Dai, Z. G., Wang, L. J., \& Wu, X. F. 2016b, ApJ, 828, 87

Wang, S. Q., Wang, L. J., Dai, Z. G., \& Wu, X. F. 2015, ApJ, 799,107

Willis, A. J. 1982, MNRAS, 198, 897

Woosley, S. E. 2010, ApJL, 719, L204

Woosley, S. E. 2018, ApJ, 863, 105

Yuan, Q., Liao, N. H., Xin, Y. L., et al. 2018, ApJL, 854, L18

Zhang, W., Woosley, S. E., \& Heger, A. 2008, ApJ, 679, 639 